\title{
Probing Extra Dimensions with ATLAS
}

\author{
Dominik Dannheim \\ Columbia University, New York; now at CERN, Experimental Physics Division, \\ CH-1211 Geneva 23, Switzerland, Email: dominik.dannheim@cern.ch
}

\begin{abstract}
In the late nineties several authors suggested that the extra dimensions predicted by string theory might lead to observable effects at high energy colliders. The ATLAS experiment which will start taking data at the LHC in 2007 will be an excellent place to search for such effects. The sensitivity of ATLAS to signatures of Extra Dimensions will be presented.
\end{abstract}

Keywords: ATLAS, LHC, Extra Dimensions, Exotics

PACS: $14.80 .-\mathrm{j}, 11.25 . \mathrm{Wx}, 13.85 . \mathrm{Qk}, 13.85 . \mathrm{Rm}$

\section{INTRODUCTION}

Over the past decade, new models based on large compactified extra spatial dimensions (ED) have been proposed [1,2], which could explain the large gap between the electroweak$(\mathrm{EW})$ and the Planck scale of $M_{E W} / M_{P l} \approx 10^{-17}$. The $1 / r$ Newtonian potential seen at large distances becomes a $1 / r^{1+n}$ potential at small distances, implying a large fundamental Newton constant when the compactification radius is large. Present direct limits on ED allow, for example, $2 \mathrm{ED}$ up to $0.13 \mathrm{~mm}$ [3]. For such distances, SM particles are constrained to reside on 3 spatial dimensions by gauge theories of EW and strong interactions, valid to $<$ fm distances, but gravitons could propagate in the bulk. The resulting fundamental scale of gravity could be as small as a few $\mathrm{TeV}$ and the observed Planck mass would not be a relevant physical scale anymore. A theoretical foundation for introducing ED is given in the framework of string theories, which need ED for self-coherence reasons.

The LHC, colliding protons at a centre-of-mass energy of $\sqrt{s}=14 \mathrm{TeV}$, will exceed the accessible mass reach of the current collider experiments by an order of magnitude into the $\mathrm{TeV}$ range. The ATLAS detector [4] at the LHC, which will become operational in 2007, will therefore be an ideal place to look for ED signatures, such as jets with missing $E_{T}$, high-mass resonances or events with a large $\Sigma E_{T}$. In the following, studies are presented which evaluate the sensitivity of ATLAS to detect signals from various ED scenarios. All results are based on a parameterised simulation model of the ATLAS detector [5]. Unless otherwise noted, an integrated luminosity of $\int L \mathrm{dt}=100 \mathrm{fb}^{-1}$ is assumed, corresponding to one year of data taking at design luminosity.

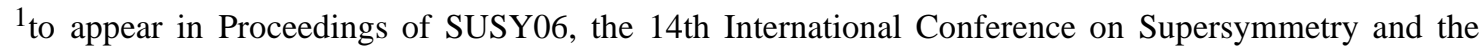
Unification of Fundamental Interactions, UC Irvine, California, 12-17 June 2006.
} 


\section{LARGE EXTRA DIMENSIONS}

In the ADD model [1], proposed by Arkani-Hamed, Dimopoulos and Dvali, the SM fields are confined to a $4 \mathrm{D}$ brane. A new gravity scale $M_{D} \approx \mathrm{TeV}$ is introduced in $4+\mathrm{n}$ dimensions. In the 4D brane, the graviton appears as a tower of closely spaced $(<1 \mathrm{meV})$ massive KaluzaKlein $(\mathrm{KK})$ excitation states with universal coupling to the SM fields. The coupling is small $\left(\approx 1 / M_{P l}\right)$, but nevertheless a sizeable cross section is obtained from the large number of interfering KK states. Virtual exchange of KK states leads to deviations in cross sections at high mass or energy and deviations in angular distributions. Direct production of gravitons results in jet + missing $E_{T}$ signatures.

ATLAS has evaluated the sensitivity to detect virtual graviton exchange in deviations in the dilepton and diphoton invariant mass spectrum [6]. The enhancement in the SM cross sections is obtained as function of an effective scale $M_{S} \approx M_{D}$. For this scenario, ATLAS will be able to probe ED up to $M_{S} \approx 7-8 \mathrm{TeV}$.

Direct graviton production with the graviton escaping undetected in the ED has been studied by searching for a possible excess of events with jets and missing energy [7]. In this scenario, ED can be probed up to $M_{D} \approx 6-9 \mathrm{TeV}$.

A variation of the ADD scenario was also considered [8], in which additional small ED orthogonal to the brane are present with a compactification radius $M_{c} \approx \mathrm{TeV}^{-1}$. Fermions are confined to the 3-brane, but gauge fields propagate in the $\mathrm{TeV}^{-1}$-scale ED. Leptonic decays of gauge excitations of the photon and the $Z$ boson would provide striking signatures at the LHC. The sensitivity of ATLAS in this scenario has been estimated to reach to values of $M_{c} \approx 5.8 \mathrm{TeV}$. A study of the angular distribution of the produced lepton pairs in the peak region showed that $\int L \mathrm{dt}=300 \mathrm{fb}^{-1}$ would be needed to distinguish such gauge excitations from mass peaks produced by SM-like $Z^{\prime}$ or graviton resonances (for $M_{c}<5 \mathrm{TeV}$ ).

\section{WARPED EXTRA DIMENSIONS}

Graviton resonances are predicted in several models with small ED. In a Randall-Sundrum model [2], the metric is not flat, but varies exponentially in an extra dimension between the $\mathrm{TeV}$ brane, on which SM particles reside, and the Planck scale. This "warped" dimension is responsible for the shrinkage of the gravitational interaction strength in our (3+1)D subspace. The boundary conditions in the ED give rise to periodic wave functions; the KK modes in $(3+1) \mathrm{D}$ for the excited graviton $G^{*}$. The mass of the first resonance is given as $m_{1} \approx$ $3.83 \Lambda_{\pi} k / \bar{M}_{P l}$, with $0.01<k / \bar{M}_{P l}<0.1$. The cross section for $G^{*}$ production is proportional to $k / \bar{M}_{P l}^{2}$. The couplings of the $G^{*}$ are universal, giving rise to many decay channels, such as $e e$, jet-jet, $\gamma \gamma, Z Z, W W$. The spin-2 nature of the $G^{*}$ can be used in a spin analysis [9] to distinguish it from SM background and from other exotic scenarios, such as $Z^{\prime}$ production.

The sensitivity for $G^{*}$ detection was studied in several of the possible decay channels [10]. The decay to two electrons is considered the most promising discovery channel. It has a low branching ratio of $\approx 2 \%$, but a very clear signature of 2 back-to-back high $p_{T}$ electrons with an invariant mass equal to the mass of the $G^{*}$. The background from SM processes is small, mostly originating from the high-mass tail of the DY spectrum. Figure 1a) shows the twoelectron invariant mass spectrum in ATLAS consisting of SM background and a hypothetical graviton resonance at $1.5 \mathrm{TeV}$ in a scenario with $k / \bar{M}_{P l}=0.01$. The signal is clearly visible 
a)

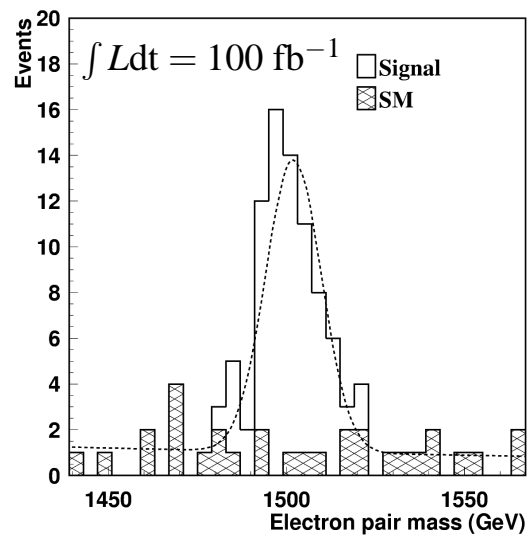

b)

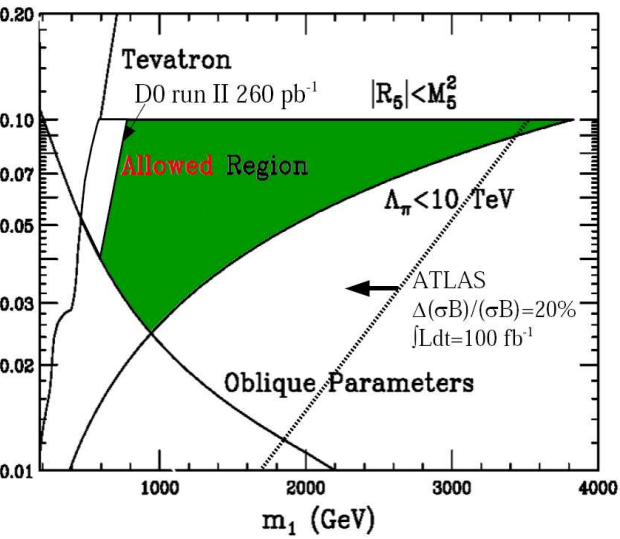

FIGURE 1. a) Invariant mass distribution for RS graviton-production at $M_{G}=1.5 \mathrm{TeV}$ and for the SM background. b) Current constraints on the RS parameter space. The expected sensitivity of ATLAS is indicated with a dashed line.

above the low SM background. The ultimate $5 \sigma$ discovery reach for $k / \bar{M}_{P l}=0.01$ was estimated to be $2080 \mathrm{GeV}$, well above the current experimental limit of $250 \mathrm{GeV}$ from the the D0 experiment at Run II of the Tevatron [11]. The currently allowed region of the RS parameter space is shown in Fig. 1b), together with the ATLAS reach for a determination of the $G^{*}$-production cross section with an accuracy of $20 \%$. The interesting region of the parameter space is covered by ATLAS.

\section{UNIVERSAL EXTRA DIMENSIONS}

Universal Extra Dimension (UED) scenarios allow all SM particles to propagate in ED of $\mathrm{TeV}^{-1}$ size. Due to tree-level KK-number conservation the KK 0-mode states can not be virtual and can only be produced in pairs. There are only weak constraints from previous electro-weak measurements. The current Tevatron results constrain the mass of the compactification scale to $M_{c}>400 \mathrm{GeV}$ [12].

For ATLAS, a study for a specific class of UED models has been performed [13]. It assumes that the topology of the UED model forms a thick brane embedded in a bulk of two large $\mathrm{ED}$, where only gravity propagates. Only models with one $\mathrm{ED}$ of $\mathrm{TeV}^{-1}$ size and only gravity-mediated decays of the KK states have been considered. The signature in this case are dijet events with large missing transverse energy. SM background from events with jets and a $W$ or a $Z$ boson was considered. At $5 \sigma$, ATLAS will be sensitive to $M_{c} \approx 2.7 \mathrm{TeV}$.

A recent phenomenological study [14] considered higher modes of KK excitations, which could be singly produced at colliders. They are heavier than the $(1,0)$ modes by a factor $\sqrt{2}$ and their cascade decays lead to a series of narrow-spaced $t \bar{t}$ resonances, resulting in lepton + jets events. Within this scenario, previous $t \bar{t}$ resonance studies have been reinterpreted. A $1 \mathrm{TeV} t \bar{t}$ resonance from UED could be observed with $\int L \mathrm{dt}=30 \mathrm{fb}^{-1}$. The reach could be further increased by taking into account extra leptons and jets produced in the cascade decays. Furthermore, if the difference of $\sqrt{2}$ between consecutive even states would be observed, one could discriminate from other scenarios and extract the number of ED. 


\section{MICRO BLACK HOLES}

At the LHC, micro black holes $(\mathrm{BH})$ could be produced if the fundamental Planck scale would be lowered to $\approx \mathrm{TeV}[15,16]$. A BH is characterised by its mass, $M_{B H}=\sqrt{s}$, its Schwarzschild radius $R_{S}=2 G M_{B H} / c^{2}$ and (for $n$ ED) by its temperature $T_{H} \approx(1+$

$n) /\left(M_{B H}^{1 /(1+n)}\right)$. The production cross section at the LHC for BHs would be about $\sigma \approx \pi R_{S}^{2} \approx$ $100 \mathrm{pb}$. BHs decay with a short lifetime of $\approx 10^{-27}-10^{-25} \mathrm{~s}$ via Hawking radiation to all degrees of freedom with equal probability. Such decays will thus result in high-multiplicity, high $\Sigma p_{T}$ spherical events with significant missing $E_{T}$. The $\mathrm{BH}$ mass can be reconstructed from the sum of all 4 momenta and the temperature can be extracted from a comparison of the energy spectrum of the final-state particles to the expectation from a black-body spectrum.

$\mathrm{BH}$ studies for ATLAS have been performed with $n=2-6 \mathrm{ED}$ and a $\mathrm{BH}$ mass from $5 \mathrm{TeV}$ up to $14 \mathrm{TeV}[17,18]$. Background from QCD events at large missing $E_{T}$ and from SUSY has been considered. It was shown that ATLAS will be able to discover higher-dimensional BHs and to explore their parameters. The $\mathrm{BH}$ parameters were extracted in a test model with $n=4$ and $M_{B H}=7 \mathrm{TeV}$ and assuming that the parton-level $\mathrm{BH}$-production cross section is known to $20 \%$. The accuracy obtained in this case was about $10 \%$ for the temperature, $15 \%$ for the mass and \pm 0.75 for the number of ED.

\section{CONCLUSIONS}

ATLAS will have an unprecedented reach for discovery of ED in the TeV range. Moreover, discrimination between models and extraction of model parameters will be possible in several scenarios.

\section{REFERENCES}

1. N. Arkani-Hammed, S. Dimopoulos and G. Dvali, Phys. Lett. B429, 263 (1998); Phys. Rev. D59, 086004 (1999).

2. L. Randall and R. Sundrum, Phys. Rev. Lett. 83, 3370 (1999); 83, 4690 (1999).

3. C.D. Hoyle et al., Phys. Rev. D70, 042004 (2004).

4. ATLAS collaboration, ATLAS Technical Design Report, CERN/LHCC 99-14/15 (1999).

5. E. Richter-Was, D. Froidevaux and L. Poggioli, ATLAS Internal Note ATL-PHYS-98,131 (1998).

6. V. Kabachenko, A. Miagkov and A. Zenin, ATLAS Internal Note ATL-PHYS-2001-12 (2001).

7. L. Vacavant and I. Hinchliffe, J. Phys., G27 no. 8, 1839 (2001).

8. G. Azuelos and G. Polesello, Eur. Phys. Journ. C, 10.1140 (2004).

9. R. Cousins et al., J. High Energy Phys. 11, 046 (2005).

10. B.C. Allanach et al., J. High Energy Phys. 9, 019 (2000); 12, 039 (2002).

11. V.M. Abazov et al., Phys. Rev. Lett. 95, 091801 (2005).

12. T. Appelquist, H.-C. Cheng and B.A. Dobrescu, Phys. Rev. D64, 035002 (2001).

13. P.-H. Beauchemin and G. Azuelos, ATLAS Internal Note ATL-PHYS-PUB-2005-03 (2005).

14. G. Burdman, B.A. Dobrescu and E. Ponton, FERMILAB-Pub-06-009-T (2006).

15. S. Giddings and S. Thomas, Phys. Rev. D65, 056010 (2002).

16. S. Dimopoulos, G. Landsberg, Phys. Rev. Lett. 87, 161602 (2001)

17. J. Tanaka et al., Eur. Phys. J. C41, 19 (2005)

18. C.M. Harris et al., J. High Energy Phys. 05, 053 (2005) 